\title{
Zur analytischen Vorzeichenbestimmung und Bereichsabschätzung von Wechselwirkungskraftkonstanten für Eigenwertprobleme der Ordnung $\boldsymbol{n}=\mathbf{3}$
}

\author{
Beispiel: Molekültyp $X Y Z$ mit $\mathbf{C}_{\mathrm{s}}$-Symmetrie \\ A. FADINI * \\ Lehrstuhl I der Technischen Mechanik der Technischen Hochschule Stuttgart \\ (Z. Naturforschg. 21 a, 2055-2057 [1966] ; eingegangen am 5. September 1966)

\begin{abstract}
Aus der Grenzwertformel $\mathbf{F}(\lambda)=\lambda \cdot \mathbf{G}^{-1}$ werden für die Ordnung $n=3$ die Vorzeichen und Zahlenwerte der Wechselwirkungsgrößen $f_{12}(\lambda), f_{13}(\lambda)$ und $f_{23}(\lambda)$ nach Formeln allgemein berechnet. Dieses Modell wird zur Vorzeichen- und Bereichsabschätzung der Wechselwirkungskraftkonstanten von hinreichend stark frequenzgekoppelten Molekülen des Typs $X Y Z$ mit $\mathbf{C}_{\mathbb{S}}$-Symmetrie verwandt. Für diesen Molekültyp $X Y Z$ mit $\mathbf{C}_{\mathrm{s}}$-Symmetrie lassen sich für Valenzwinkel $a$ mit $90^{\circ} \leqq a<180^{\circ}$ vollständig analytische Vorzeichenregeln aufstellen, die keine zusätzlichen numerischen Auswertungen erfordern. Zur Veranschaulichung dieser Vorzeichenregeln und Bereichsabschätzungen dienen die Moleküle $\mathrm{HOCl}, \mathrm{DOCl}$ und NOF als Zahlenbeispiele.
\end{abstract}

\section{Allgemeine Vorzeichenregeln und Bereichsabschätzungen für $n=3$}

Für Probleme der Ordnung $n=3$ läßt sich die Grenzwertformel ${ }^{1}$

$$
\mathbf{F}(\lambda)=\lambda \mathbf{G}^{-1}
$$

noch übersichtlich anschreiben. Dabei ist $\lambda$ der Eigenwert, $\mathbf{G}^{-1}$ die Matrix der kinetischen Energie und $\mathbf{F}(\lambda)$ die zu einer Lösung zusammengeschrumpfte Lösungsmannigfaltigkeit der durch die Säkulargleichung definierten Kraftkonstantenmatrix F. Da bei der praktischen Kraftkonstantenrechnung im allge- meinen die inverse Matrix G der kinetischen Energie gegeben ist, muß die zu

$$
\mathbf{G}=\left(\begin{array}{lll}
g_{11} & g_{12} & g_{13} \\
g_{12} & g_{22} & g_{23} \\
g_{13} & g_{23} & g_{33}
\end{array}\right)
$$

inverse Matrix $\boldsymbol{G}^{-1}$ nach den Regeln der Matrizenrechnung ${ }^{2}$ aufgestellt werden. Die hierfür notwendige Voraussetzung

$$
\operatorname{det} \mathbf{G}>0
$$

ist wegen der positiven Definitheit der Matrix $\mathbf{G}$ als inverse Matrix der kinetischen Energie erfüllt ${ }^{3,4}$. Wir erhalten

$$
\mathbf{F}(\lambda)=\lambda \operatorname{det}^{-1} \mathbf{G}\left(\begin{array}{ccc}
g_{22} g_{33}-g_{23}{ }^{2} & -\left(g_{12} g_{33}-g_{13} g_{23}\right) & g_{12} g_{23}-g_{13} g_{22} \\
-\left(g_{12} g_{33}-g_{13} g_{23}\right) & g_{11} g_{33}-g_{13}{ }^{2} & -\left(g_{11} g_{23}-g_{12} g_{13}\right) \\
g_{12} g_{23}-g_{13} g_{22} & -\left(g_{11} g_{23}-g_{12} g_{13}\right) & g_{11} g_{22}-g_{12}{ }^{2}
\end{array}\right) .
$$

Aus Gl. (4) entnehmen wir für die Wechselwirkungsgrößen

$$
\begin{aligned}
& f_{12}(\lambda)=\lambda \operatorname{det}^{-1} \mathbf{G}\left(g_{13} g_{23}-g_{12} g_{33}\right), \\
& f_{13}(\lambda)=\lambda \operatorname{det}^{-1} \mathbf{G}\left(g_{12} g_{23}-g_{13} g_{22}\right), \\
& f_{23}(\lambda)=\lambda \operatorname{det}^{-1} \mathbf{G}\left(g_{12} g_{13}-g_{11} g_{23}\right) .
\end{aligned}
$$

Da $\lambda$ als Eigenwert stets positiv ist und $\operatorname{det} \mathbf{G}$ wegen (3) stets größer Null ist, ergeben sich für die Wechselwirkungsgrößen $f_{12}(\lambda), f_{13}(\lambda)$ und $f_{23}(\lambda)$ die Vorzeichenregeln

\footnotetext{
* Privatadresse: 74 Tübingen, Breuningstr. 31.

1 A. F AdinI, Z. Naturforschg. 21 a, 484 [1966].

2 R. Zurmühl, Matrizen, Springer-Verlag, Berlin 1961, S. 35 bis 37 .

3 Siehe ${ }^{2}$, S. 14, 33, 129-134.
}

$$
\begin{aligned}
& \operatorname{sign} f_{12}(\lambda)=\operatorname{sign}\left(g_{13} g_{23}-g_{12} g_{33}\right), \\
& \operatorname{sign} f_{13}(\lambda)=\operatorname{sign}\left(g_{12} g_{23}-g_{13} g_{22}\right), \\
& \operatorname{sign} f_{23}(\lambda)=\operatorname{sign}\left(g_{12} g_{13}-g_{11} g_{23}\right) .
\end{aligned}
$$

Die Gln. (5) bis (7) und (8) bis (10) dienen als Modell zur numerischen Bereichsabschätzung und Vorzeichenbestimmung der Wechselwirkungskraftkonstanten hinreichend stark frequenzgekoppelter Moleküle ${ }^{1,5,6}$.

4 F. Matossi, Gruppentheorie der Eigenshwingungen von Punktsystemen, Springer-Verlag, Berlin 1961, S. 120.

5 A. Fadini, Fortschritte in der Raman-Spektroskopie, Magdeburg, 11.-14. Oktober 1966, Vortragsauszüge.

6 A. Fadini, Z. Angew. Math. Mech. 46 (Sonderheft) [1966] (im Druck). 


\section{Bereichsabschätzung und Vorzeichenregeln für den Molekültyp XYZ mit $\mathrm{C}_{\mathrm{s}}$-Symmetrie}

Nach $\mathrm{W}_{\text {ILson }}{ }^{7}$ lautet die Matrix $\mathbf{G}$ für den Mole kültyp $X Y Z$ mit der Symmetrie $\mathbf{C}_{\mathrm{s}}$

$$
\mathbf{G}=\left(\begin{array}{ccc}
\mu_{X}+\mu_{Y} & \mu_{Y} \cos \alpha & -\mu_{Y} r \bar{Y}^{-1} \sin \alpha \\
\mu_{Y} \cos \alpha & \mu_{Y}+\mu_{Z} & -\mu_{Y} r_{\bar{X}}^{1} \sin \alpha \\
-\mu_{Y} r_{Y Z}^{-1} \sin \alpha & -\mu_{Y} r_{X Y}^{-1} \sin \alpha \frac{\mu_{X}}{r_{X Y}^{2}}+\frac{\mu_{Z}}{r_{Y Z}^{2}}+\mu_{Y}\left(r_{X Y}^{-2}+r \overline{Y Z}^{2}-\frac{2 \cos \alpha}{r_{X Y} r_{Y Z}}\right)
\end{array}\right) .
$$

(Dabei sind $\mu_{X}, \mu_{Y}$ und $\mu_{Z}$ die reziproken Massen der Atome $X, Y$ und $Z, r_{X Y}$ und $r_{Y Z}$ die Gleichgewichtsabstände zwischen den Atomen $X$ und $Y$ bzw. $Y$ und $Z$, und $\alpha$ ist der Valenzwinkel zwischen $X Y Z$.)

Die Wechselwirkungsgrößen erhalten wir aus den Gln. (11), (2), (5), (6) und (7) zu

$$
\begin{gathered}
f_{12}(\lambda)=\lambda \operatorname{det}^{-1} \mathbf{G}\left\{\left(1+\cos ^{2} \alpha\right) \mu_{Y}^{2} r_{X Y}^{-1} r_{Y Z}^{-1}\right. \\
\left.-\mu_{Y} \cos \alpha\left[\mu_{X} r_{X Y}^{-2}+\mu_{Z} r_{Y Z}^{-2}+\mu_{Y}\left(r_{X}^{-2}+r_{Y Z}^{-2}\right)\right]\right\}, \\
f_{13}(\lambda)=\lambda \operatorname{det}^{-1} \mathbf{G} \mu_{Y} \sin \alpha\left[r_{Y Z}^{-1}\left(\mu_{Y}+\mu_{Z}\right)\right. \\
\left.\quad-\mu_{Y} r_{X Y}^{-1} \cos \alpha\right], \\
f_{23}(\lambda)=\lambda \operatorname{det}^{-1} \mathbf{G}\left\{\left[\left(\mu_{X}+\mu_{Y}\right) r_{X Y}^{-1}\right.\right. \\
\left.\left.-\mu_{Y} r_{Y Z}^{-1} \cos \alpha\right] \mu_{Y} \sin \alpha\right\} .
\end{gathered}
$$

Daraus ergeben sich nach den Gln. (8), (9) und (10) und wegen $\mu_{Y}>0$ sofort die allgemeinen Vorzeichenregeln

$$
\begin{aligned}
& \operatorname{sign} f_{12}(\lambda)=\operatorname{sign}\left\{\left(1+\cos ^{2} \alpha\right) \mu_{Y} r_{X Y}^{-1} r_{Y Z}^{-1}\right. \\
& \left.\quad-\cos \alpha\left[\mu_{X} r_{\bar{X}}^{-2} \bar{Y}+\mu_{Z} r_{\bar{Y}}^{-2}+\mu_{Y}\left(r_{\bar{X}}^{-2}+r_{\bar{Y}}-2\right)\right]\right\},
\end{aligned}
$$$$
\operatorname{sign} f_{\mathbf{1 3}}(\lambda)=\operatorname{sign}\left\{\left[r_{\mathrm{Y} Z}^{-1}\left(\mu_{Y}+\mu_{Z}\right)\right.\right.
$$$$
\left.\left.-\mu_{Y} r_{X Y}^{-1} \cos \alpha\right] \sin \alpha\right\},
$$

$\operatorname{sign} f_{23}(\lambda)=\operatorname{sign}\left\{\sin \alpha\left[\left(\mu_{X}+\mu_{Y}\right) r_{X Y}^{-1}\right.\right.$

$$
\left.\left.-\mu_{Y} \cos \alpha r_{\mathrm{Y} Z}^{-1}\right]\right\} \text {. }
$$

\section{Vorzeichenregeln für $X Y Z$ mit $\mathrm{C}_{\mathrm{s}}$-Symmetrie für Valenzwinkel $\alpha \geqq 90^{\circ}$}

Für einen Valenzwinkel $\alpha$ mit

$$
90^{\circ} \leqq \alpha<180^{\circ} 7 \mathrm{a}
$$

folgen aus den Gln. (15), (16) und (17) die drei einfachen Vorzeichenregeln

$f_{12}(\lambda)>0, \quad f_{13}(\lambda)>0, \quad f_{23}(\lambda)>0$.

7 E. B. Wilson, J. C. Decius u. P. C. Cross, Molecular Vibrations, Verlag McGraw-Hill, New York 1955, S. 63.

7a Im Falle $\alpha=180^{\circ}$ gehört $X Y Z$ zur Symmetriegruppe $\mathbf{C}_{\infty} \mathrm{v}$. Hierfür ist $f_{13}=f_{23}=0$. Aus Gl. (15) folgt die Vorzeichenregel $f_{12}(\lambda)>0{ }^{1,6}$.

8 Für den Valenzwinkel $a$ mit $0^{\circ}<a<90^{\circ}$ müßten die Vorzeichenregeln (15), (16) und (17) numerisch ausgewertet werden.
Für diesen Fall der Valenzwinkel können also die Vorzeichen aller 3 Wechselwirkungsgrößen vollständig analytisch, d. h. ohne zusätzliche numerische Auswertung der Formeln, bestimmt werden. Diese Tatsache ist für die praktische Kraftkonstantenrechnung ein günstiger Umstand ${ }^{8}$, da die Valenzwinkel der bekannten Moleküle des Typs $X Y Z$ mit $\mathbf{C}_{\mathrm{s}}$-Symmetrie die Bedingung (18) erfüllen ${ }^{9}$. Verwenden wir hiermit die Gln. (19), (20) und (21) als Modell für die Vorzeichenbestimmung der Wechselwirkungskraftkonstanten realer Moleküle, so erwarten wir bei hinreichend starken Frequenzkopplungen ${ }^{6}$ lauter positive Wechselwirkungskraftkonstanten.

\section{Beispiele zu den Vorzeichenregeln für $\alpha \geqq 90^{\circ}$}

Als Beispiele zur praktischen Veranschaulichung der Vorzeichenregeln (19), (20) und (21) für Molekültypen $X Y Z$ mit Valenzwinkeln größer als $90^{\circ}$ wählen wir die 3 Moleküle HOCl, DOCl und ONF aus. Für die Moleküle HOCl und DOCl sind aus Isotopenmessungen die Wechselwirkungskraftkonstanten $f_{\mathrm{OCl} / \alpha}$ bekannt ${ }^{10}$. Für das Molekül ONF liegen Berechnungen für sämtliche Wechselwirkungskraftkonstanten ${ }^{11}$ nach dem Kopplungsstufenverfahren (s. Anm. ${ }^{12,13}$ ) vor. Die in Tab. 1 eingetragenen Zahlenwerte zeigen eine schöne Übereinstimmung mit den Vorzeichenregeln (19) bis (21).

Es sei weiterhin vermerkt, daß auch für die weiteren Moleküle $\mathrm{O}^{13} \mathrm{NCl}, \mathrm{O}^{15} \mathrm{NCl}, \mathrm{ONBr}$ und $\mathrm{NSF}$ sämtliche, nach dem Kopplungsstufenverfahren berechneten Wechselwirkungskraftkonstanten positiv sind $^{11}$, obwohl hier bereits recht schwache Kopplungen vorliegen.

9 Siehe z. B. H. Siebert, Anwendungen der Schwingungsspektroskopie in der Anorganischen Chemie, Springer-Verlag, Berlin 1966, S. 52. (Vergleichsweise siehe auch S. 50 für $\mathrm{XY}_{2}$-Molekültypen!)

10 K. Hedberg u. R. M. Badger, J. Chem. Phys. 19, 508 [1951].

11 W. Sawodny, A. Fadini u. K. Ballein, Spectrochim. Acta 21, 995 [1965].

12 A. F Adini, Z. Angew. Math. Mech. 44, 506 [1964].

13 A. Fadini, Z. Angew. Math. Mech. 45, T 29 [1965]. 


\begin{tabular}{|c|c|c|c|}
\hline $\begin{array}{c}\text { Wechselwirkungs- } \\
\text { kraftkonstanten }\end{array}$ & \multicolumn{3}{|c|}{ Molekül $X Y Z$} \\
\hline$f_{i k}$ für $i \neq k$ & HOCl & DOCl & ONF \\
\hline$f_{12}=f_{X Y / Y Z}$ & & & 0,12 \\
$f_{13}=f_{X Y /_{a}} r X Y$ & 0,51 & 0,51 & 0,22 \\
$f_{23}=f_{Y Z} /_{a} r Y Z$ & 0,41 \\
\hline
\end{tabular}

Tab. 1. Die bekannten Wechselwirkungskraftkonstanten der Moleküle HOCl, DOCl ${ }^{10},{ }^{14}$ und $\mathrm{ONF}^{11}$ in mdyn/ $/$.

\section{Lösungsbereiche der Wechselwirkungskraft- konstanten für HOCl}

Da für das Molekül ONF die Lösungsbereiche der Wechselwirkungskraftkonstanten bereits angegeben worden sind ${ }^{5}$, beschränken wir uns auf das Molekül $\mathrm{HOCl}$.

Wegen der schwachen Kopplungen verwenden wir zur Berechnung der Wechselwirkungsgröße $f_{i k}(\lambda)$ mit $i \neq k$ für den Eigenwert $\lambda$ stets den größeren der beiden experimentellen Eigenwerte $\lambda_{i}$ bzw. $\lambda_{k}{ }^{5}$. Da weiterhin die G-Matrix unter Zugrundelegung der in ${ }^{10}$ angegebenen Ausgangsdaten numerisch vorgelegen ist, sind die Bereiche aus den Gln. (5), (6) und (7) berechnet worden. Als Ergebnisse erhalten wir:

$$
\begin{gathered}
0<f_{12}=f_{\mathrm{HO} / 0 \mathrm{Cl}}<2,2 \mathrm{mdyn} / \AA, \\
0<f_{13}=f_{\mathrm{H} O / a} r_{\mathrm{HO}}<0,32 \mathrm{mdyn} / \AA, \\
0<f_{23}=f_{\mathrm{OCl} / a} r_{\mathrm{OCl}}<0,53 \mathrm{mdyn} / \AA .
\end{gathered}
$$

Der Literaturwert für $f_{23}=0,51 \mathrm{mdyn} / \AA$ liegt an der oberen Grenze des Abschätzungsintervalles.

\section{Weitere Anwendungen}

Für die Eigenwertprobleme der Ordnung $n=3$ der Moleküle der Typen und Symmetrien $X Y_{2} Z\left(\mathbf{C}_{2 \mathrm{v}}\right)$, $X Y_{5}\left(\mathbf{D}_{3 \mathrm{~h}}\right), X Y_{5}\left(\mathbf{C}_{4 \mathrm{v}}\right), X Y_{3} Z\left(\mathbf{C}_{3 \mathrm{v}}\right)$ und anderer $\mathrm{mehr}$ können in entsprechender Weise Vorzeichenregeln und Formeln zur Bereichsabschätzung aufgestellt werden.

Den Herren Prof. J. Goubeau, Prof. A. Slibar, Prof. H. J. Becher und Prof. O. Glemser möchte ich herzlich für ihr Interesse und ihre Unterstützung danken.

${ }^{14}$ Die angegebenen Kraftkonstanten $f_{23}$ für $\mathrm{HOCl}$ sind nach ${ }^{10}$ aus 2 Datensätzen gemittelt. 